\title{
Congenital Hypothyroidism: Incidence, Etiology and Laboratory Screening
}

\author{
Liong Boy Kurniawan \\ Department of Clinical Pathology, Faculty of Medicine, Hasanuddin University/Dr. Wahidin Sudirohusodo, Makassar, Indonesia. E-mail: \\ liongboykurniawan@yahoo.com
}

\begin{abstract}
Congenital hypothyroidism is a condition resulting from a deficiency of thyroid hormone in newborns. Congenital hypothyroidism has no specific signs and symptoms at birth. It may lead to severe mental retardation and growth, and developmental disorders. Therefore, it is essential to perform newborn laboratory screening tests for prompt diagnosis and treatment to minimize the sequels. Laboratory screening tests are performed by taking prick blood from the heel of newborn and testing either TSH or T4 or both of them. Currently, the congenital hypothyroidism screening is not mandatory in Indonesia, but some multicentered screening programs have been performed. In Indonesia, a TSH level above $20 \mu \mathrm{U} / \mathrm{mL}$ is used as a cutoff that needs a confirmatory test using serum samples to confirm congenital hypothyroidism diagnosis. Once the diagnosis is established, prompt treatment and laboratory monitoring are needed for a better outcome.
\end{abstract}

Keywords: Congenital hypothyroidism, laboratory, screening

\section{INTRODUCTION}

Hypothyroidism term refers to an endocrine disorder resulting from the lack or deficiency of thyroid hormone (thyroxin) and effecting peripheral tissues. ${ }^{1}$ Congenital Hypothyroidism $(\mathrm{CH})$ can be simply defined as deficiency of thyroid hormone present at birth. ${ }^{2}$ Congenital hypothyroidism is a common term for deficiency of thyroid hormone due to dysfunction of the thyroid gland or abnormality in thyroid gland morphology which develops during the fetal or perinatal stage. ${ }^{3}$ It is one of the most common conditions in neonates which may lead to mental retardation. It is preventable if the diagnosis and treatment are not performed in a few weeks after birth. Most of the neonates with $\mathrm{CH}$ do not have clear hypothyroidism manifestations making the diagnosis a challenge. Infants have some residual thyroid hormone that the symptoms and signs of thyroid hormone deficiency are not specific. In the condition of fetal total thyroid hormone deficiency due to the absence of production/secretion, thyroid hormone derived from maternal transplacental passage still provide protection until birth. It is estimated that one-third of thyroxin (T4) from the mother crosses to the fetus during pregnancy. Maternal T4, which has a half-life of six days, will be metabolized and excreted at the age of three or four weeks. If $\mathrm{CH}$ is left untreated within the first few weeks or months, the symptoms will be worsening and will cause a disturbance in brain development. Due to this, it is urgent and important to screen, diagnose $\mathrm{CH}$, and begin $\mathrm{CH}$ treatment immediately after birth. The best way to prevent late-diagnosis of $\mathrm{CH}$ is by performing large population screening in newborns. ${ }^{4}$

\section{Epidemiology}

Before the era of newborn screening programs started, the occurrence of $\mathrm{CH}$ was diagnosed based on delayed clinical findings between 1:7,000 to $1: 10,000 .^{5}$ Following the time of large population screening, the incidence was reported to increase between $1: 3,000$ to $1: 4,000 .^{6}$ Unfortunately, Indonesia still does not have the national data of $\mathrm{CH}$. In Indonesia, data of $\mathrm{CH}$ could be obtained from Dr.Cipto Mangunkusumo Hospital in Jakarta, and Hasan Sadikin Hospital in Bandung. Through 2000 to September 2014, there were $85 \mathrm{CH}$ positive (incidence 1:2,513) from 213,669 newborns that were screened, which were higher than the reported global incidence $(1: 3,000){ }^{7}$ Medical records evaluation in Dr.Cipto Mangunkusumo Hospital and Hasan Sadikin Hospital showed more than $70 \%$ of $\mathrm{CH}$ cases were diagnosed at the age older than one year with permanent mental deficit state. Only 2.3\% of the cases were diagnosed at the age younger than three months, who had minimal growth and developmental disorders. ${ }^{7.8}$ 


\section{Etiology}

The failure of thyroid gland development (thyroid dysgenesis) is the main cause of permanent primary congenital hypothyroidism. It occurs sporadically, contributing around $85 \%$ of $\mathrm{CH}$ cases. Two-thirds of thyroid dysgenesis caused by ectopic thyroid gland location, with minor cases caused by aplasia or hypoplastic gland. The exact etiology for most of thyroid dysgenesis cases remain unclear. Some genetic mutations in genes related to transcription factors that regulate the development of thyroid glands such as thyroid transcription factor 2 (TTF-2), NKX2, PAX8 may explain these defects. However, only $2 \%$ of dysgenesis cases were reported to have these kinds of genetic mutation. ${ }^{4,9}$

Another main cause of permanent primary congenital hypothyroidism is thyroid hormone synthesis/production defect, which is termed as thyroid dyshormogenesis that contributes nearly $15 \%$ of the cases. These defects are inherited through autosomal recessive pattern and related to genes mutations that coded the Sodium-Iodine Symporter (NIS), Thyroid Peroxidase (TPO), hydrogen peroxide generation such as Thyroid Oxidase (THOX) and Dual Oxidase Maturation Factors (DUOXA), Thyroglobulin $(\mathrm{Tg})$ and iodotyrosine deiodinase. ${ }^{4,9}$

Secondary or central congenital hypothyroidism is commonly caused by isolated Thyroid-Stimulating Hormone (TSH) deficiency or congenital hypopituitarism (multiple pituitary hormone deficiencies). As for the peripheral congenital hypothyroidism, it can be caused by thyroid hormone transport defect (monocarboxylate transporter 8), thyroid hormone metabolic defect (selenocysteine insertion sequence binding protein 2) or thyroid hormone resistance. ${ }^{4}$

Transient congenital hypothyroidism may occur in several conditions such as; maternal or neonatal excess iodine exposure, maternal or neonatal iodine deficiency, maternal consumption of antithyroid drugs, a maternal antibody which block TSH receptor, THOX2 or DUOXA2 heterozygous mutations or congenital hepatic hemangioma.4 Mothers with Grave's disease who ingest antithyroid drugs may inhibit the synthesis of fetal thyroid hormone that can occur from several days to 2 weeks after birth. ${ }^{3}$ Maternal antithyroid antibodies may cross through the placenta resulting in the blocking of thyroid TSH receptors in neonatal. This situation can last between three to six months after birth as the maternal antibody levels decline. ${ }^{2}$ Etiology of congenital hypothyroidism are shown in Table 1.

Table 1. Etiology of congenital ${ }^{4}$

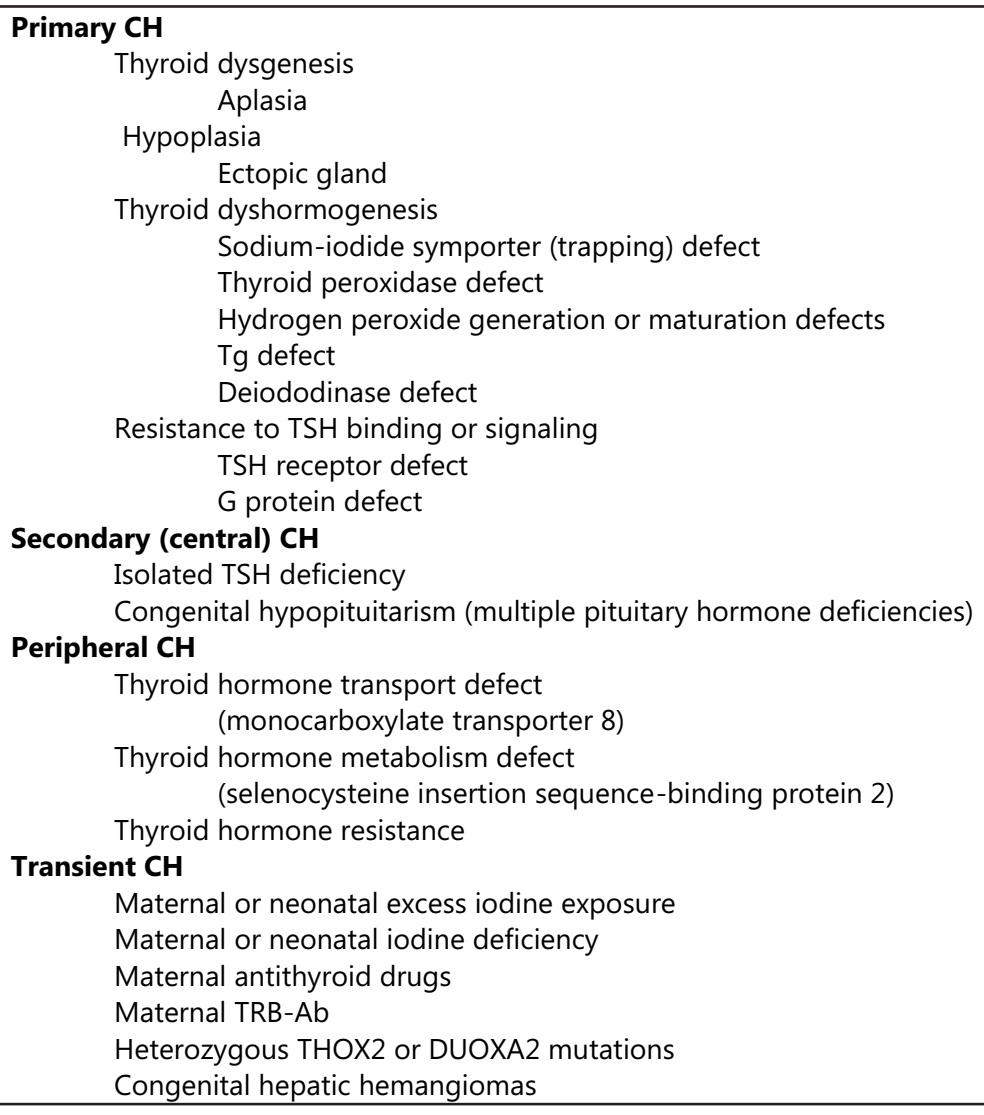




\section{Clinical Manifestation of Congenital Hypothyroidism: Symptoms and Signs}

Most of the newborns with congenital hypothyroid have no marked clinical signs and symptoms. Only 5 to $10 \%$ of them have shown the manifestation of the symptoms at birth or immediately after birth. The majority of the newborns will appear normal and healthy that the clinician may not suspect them with $\mathrm{CH}^{10}{ }^{10}$ This is might due to the maternal thyroid hormone that travels from the placenta to the fetus. This hormone may provide a transient protective effect in newborns. ${ }^{2}$ Severe congenital hypothyroid baby who gets no treatment in four to six weeks may seem to be healthy with prolonging sleeping time, not awakening for feed, slow feeding, lethargy, constipation, hoarse cry or excessive sleeping. The physical examination may show macroglossia, wide fontanels, umbilical hernia, prolong jaundice, skin mottling and dry skin (Figure 1). ${ }^{2,11}$

\section{DIAGNOSIS}

In most countries that provide newborn screening programs policy such as the United States, Canada, Japan, Australia, New Zealand, Western Europe, the diagnosis of congenital hypothyroidism is performed by the newborn screening test. While neonates in other non-provider countries, the establishment of diagnosis is usually late, after clinical manifestations of hypothyroidism already occur. ${ }^{2,4}$

The timing for the collection of the specimen is crucial to have the best diagnosis. Reported that the best time to collect a specimen for congenital hypothyroid testing is in 72 hours of age (normal newborn). This is the average time needed by the metabolism of the newborn to adapt, stabilize, and adjust to the new environment after birth. If TSH is the chosen screening test, it is important to collect the specimen after 24 hours of birth to minimize the false positive number. This false-positive number may happen due to the physiological of TSH surge in the first hour after birth. If mass screening must be performed before 24 hours of age, then the T4 test is the best choice because it is relatively more stable during this period. ${ }^{12}$

The chosen specimen for the newborn screening test is blood pricking. The blood sample is taken from the newborn's heel and collected on standard paper

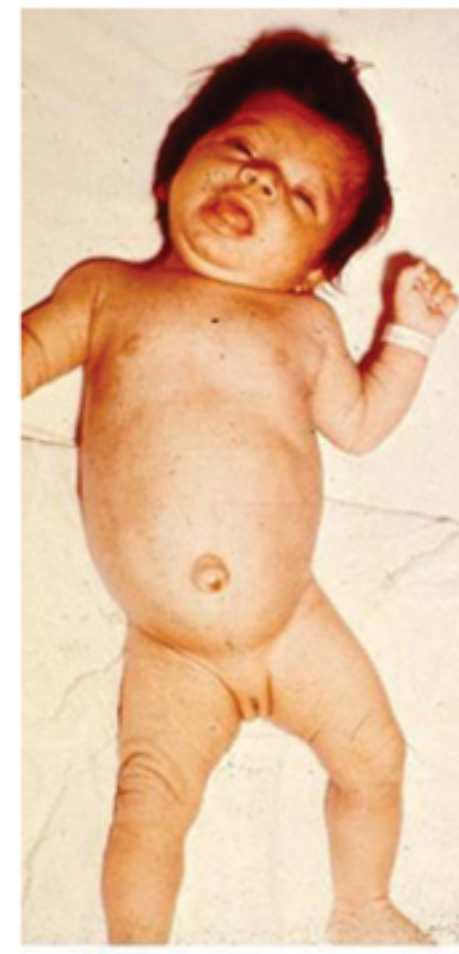

A

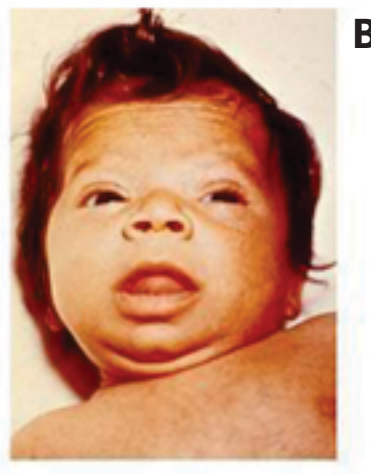

B

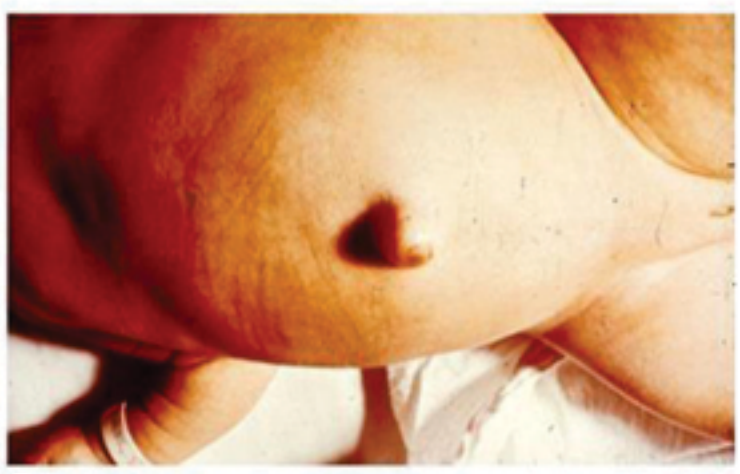

C

Figure 1. An infant with congenital hypothyroidism. A. 3-month-old infant with untreated $\mathrm{CH}_{\text {; }}$ picture demonstrates hypotonic posture, myxedematous facies, macroglossia, and umbilical hernia. B. The same infant, close up of face, showing myxedematous facies, macroglossia, and skin mottling. C. The same infant, close up showing abdominal distension and umbilical hernia. ${ }^{2}$ 
cards. Most of the specimens are collected between two and five days after born. The specimen is collected before the baby is being discharged. The filter paper cards are sent to the reference laboratory for the thyroid hormones test. ${ }^{2}$ There are three common testing protocols including TSH or T4 as the primary screen, or both T4 and TSH. As for the primary screening of congenital hypothyroid, it depends on the ability of the program in obtaining blood specimens. In the United States, the newborns are mostly being discharged from the hospital within the first 24 hours after birth. The T4 test was being picked as the screening method. In other countries such as Japan and Europe, if the hospital discharge occurs later, the selected screening method is $\mathrm{TSH}^{12}$

Congenital hypothyroidism testing is not affected by the baby's diet. If the newborn is to receive a blood transfusion, the specimen must be collected before the transfusion takes place. Instead, if the specimen cannot be obtained beforehand, the collection of the specimen must be done four months after the recent transfusion. This is to avoid getting false-negative result due to the blood mixing between the baby and the donor. ${ }^{12}$

Newborns with the specific condition have a risk to develop transient congenital hypothyroidism. They are the premature neonates (gestation age less than 37 weeks), infants with low birth weight $(<2000 \mathrm{~g})$, and hospitalized babies in a Neonatal Intensive Care Unit (NICU). Among infants with low birth weight and neonates in NICU, the first blood sampling can be obtained at the age of four to six days. Although the first screening at age of four to six days was done and shown ordinary result, the second screening must be performed in premature neonates and one-month-old baby with low birth weight, the bodyweight reaches $2,500 \mathrm{~g}$ or is discharged from hospital. ${ }^{3,8}$

While if TSH is used as primary screening, most programs use cutoff $20-35 \mu \mathrm{U} / \mathrm{mL}$, depending on the reagents and method used to determine the risk of newborns having congenital hypothyroidism. ${ }^{12}$ On the contrary, if $\mathrm{T} 4$ is used as primary screening, most programs use initial $\mathrm{T} 4<10^{\text {th }}$ percentile as the cut-off to have additional follow up TSH test. ${ }^{2,12}$ In one report, by decreasing the TSH cut-off point lower to 6 and $10 \mu \mathrm{U} / \mathrm{mL}$, a significant number of children with true and transient congenital hypothyroidism is diagnosed. ${ }^{13}$

In Indonesia, TSH cut-off $20 \mu \mathrm{U} / \mathrm{mL}$ is used. When the test shows the TSH level is below $20 \mu \mathrm{U} / \mathrm{mL}$, the test is considered normal. Then, the result must be delivered within seven days. In contrast, if the TSH level is $>20 \mu \mathrm{U} / \mathrm{mL}$ (considered high), the resampling or Duplo test must be performed. Lastly, if the TSH level is $>20 \mu \mathrm{U} / \mathrm{mL}$, confirmatory TSH and T4 serum must be performed to establish the diagnosis. ${ }^{8}$

\section{Confirmatory Serum Thyroid Test}

If an infant is detected with abnormal thyroid hormones during the screening test, a confirmatory serum thyroid test must be performed as soon as possible by recalling the patient and obtaining a venipuncture blood sample for the test. Thyroid-stimulating hormone and the confirmatory test either free T4 or total T4 must be done. ${ }^{2}$

Once the confirmatory tests have been performed, it is essential to compare the results with the appropriate reference ranges according to the baby's age. In the early few days after born, serum TSH can rise as high as $39 \mu \mathrm{U} / \mathrm{mL}$ due to the surge of TSH during this time. Most confirmatory serum tests are performed at the age of one to two weeks when the upper range of TSH falls around ten $\mu \mathrm{U} / \mathrm{mL}$. $^{2}$ The thyroid function tests (free T4, Total T4, TSH) reference age range from one to four days and two to four weeks are shown in Table 2..$^{14}$ All thyroid hormones levels are higher in one to four days after born. However, at the age of two to four weeks, the levels fall closer to the reference typically seen in infancy. ${ }^{2}$

If the serum TSH level is elevated and free T4 or total T4 is low, then the primary hypothyroidism is established. If the serum TSH level is elevated, but the free T4 or total T4 is within the normal range, primary subclinical hypothyroidism is confirmed. This is because the development of the brain is dependent on the optimal level of thyroid hormone. In most cases, treating infants with subclinical hypothyroidism is recommended. ${ }^{2}$ In most preterm infants or acutely ill infants with primary

Table 2. Reference ranges for thyroid function tests at ages 1-4 days and 2-4 weeks ${ }^{14}$

\begin{tabular}{cccc}
\hline Age & Free T4 (pmol/L) & $\begin{array}{c}\text { Total T4 } \\
(\mathbf{n m o l} / \mathbf{L})\end{array}$ & TSH (mU/L) \\
\hline 1-4 days & $25-64$ & $129-283$ & $<39$ \\
2-4 weeks & $10-26$ & $90-206$ & $<10$ \\
\hline
\end{tabular}


Table 3. The schedules for thyroid tests during $\mathrm{CH}$ treatment $^{8}$

At two weeks after starting with levothyroxine treatment

Every four weeks until reaching a normal TSH level

Every two months until the infant reaches the age of 12 months

At the age of 1 to 3 year, clinical and laboratory tests must be performed every four months

Later, the laboratory tests are performed every six months until growth is complete

After reaching 18 years old, the monitoring is under the responsibility of internist

Laboratory tests must be performed earlier if it is doubtful that the patient does not take, the treatment

regularly or when the dosage is adjusted (4-6 weeks after dosage adjustment)

Table 4. Targets for $\mathrm{TSH}, \mathrm{T} 4$ and $\mathrm{fT} 4$ levels in the first year of life during $\mathrm{CH}$ treatment $\mathrm{t}^{2,8}$

Serum free T4 or total T4 must be kept in the upper range of normal level

Serum T4 within $130-206 \mathrm{nmol} / \mathrm{L}(10-16 \mu \mathrm{g} / \mathrm{dL})$

Serum fT4 within $18-30 \mathrm{pmol} / \mathrm{L}(1.4-2.3 \mathrm{ng} / \mathrm{dL})$

Serum TSH must be kept under $5 \mathrm{mU} / \mathrm{L}$

hypothyroidism, the elevation of TSH level on the first screening test might not be observed. Hence, the second screening test is obligatory. ${ }^{14}$ Once congenital hypothyroidism is diagnosed, the treatment with levothyroxine must follow. ${ }^{2,4}$

\section{Monitoring TSH and T4/fT4 Levels}

During the treatment, TSH and T4/fT4 levels monitoring are needed for levothyroxine dosage adjustment. The schedules for thyroid hormones tests are shown in Table 3 as follow:

Targets for TSH, T4 and fT4 levels in the first year of life during $\mathrm{CH}$ treatment are shown in Table $4 .{ }^{2.8}$

\section{PROGNOSIS}

Thyroid hormone is essential for normal brain growth and development, myelination, and normal neuronal connections. The first few months afterbirths are the most critical period for the thyroid hormone to induce brain growth and development. Therefore, early detection and treatment are crucial. ${ }^{15}$ Mental disabilities in congenital hypothyroidism are characterized by poor quality of life, low self-esteem, and retarded socialization. Most of the characters are caused by Intelligence Quotient (IQ) deficiency. ${ }^{16}$

Before the newborn screening era, the diagnosis of congenital hypothyroidism was late and diagnosed after significant development occurred. It was reported that there is an inverse relationship between the age of diagnosis and IQ score. Research from Pittsburgh Children's Hospital reported that if the thyroid hormone replacement started between birth and the age of three months, the IQ mean is 89; while if the treatment started between the age of three to six months, the mean IQ is 71 ; and if the treatment did not start after the age of six months, the mean IQ is dropped till $54 .{ }^{17}$

After newborn screening programs were introduced in the mid-1970s, earlier diagnose and treatment in infants with congenital hypothyroidism were performed. Significant success in achieving better neurocognitive development was gained. One review of 51 published reports on the infants'IQ with congenital hypothyroidism compared to normal sibling or classmate was reported. This review concluded that 18 studies reported no significant IQ difference, while 33 reported a significant five to 25 lower IQ points in congenital hypothyroidism subjects. $^{18}$

\section{REFERENCES}

1. Kostic I, Curcio F. Cause of hypothyroidism, hypothyroidism-influences and treatments, Croatia, Dr. Drahomira Springer (Ed), InTech, 2012; 151-166.

2. Rastogi MV, LaFranchi SH. Congenital hypothyroidism. Orphanet Journal of Rare Diseases, 2010; 5: 17.

3. Nagasaki K, Minamitani K, Anzo M, Adachi M, Ishii T, Onigata $\mathrm{K}$, et al. Guidelines for mass screening of congenital hypothyroidism (2014 revision). Clin Pediatr Endocrinol, 2015; 24(3): 107-133.

4. LaFranchi. Approach to the diagnosis and treatment of neonatal hypothyroidism. J Clin Endocrinol Metab, 2011; 96(10): 2959-2967.

5. Alm J, Larsson A, Zetterstrom R. Congenital hypothyroidism in Swede, incidence and age at diagnosis. Acta PaediatrScand, 1978; 67(1): 1-3.

6. Fisher DA. Second international conference on neonatal thyroid screening: Progress Report. Jpediatr, 1983; 102(5): 653-654. 
7. Infodatin Kementerian Kesehatan RI. Situasi dan analisis penyakit tiroid. 2015; 1-7.

8. Kementerian Kesehatan Republik Indonesia. Pedoman skrining hipotiroid kongenital. 2014; 36-41.

9. Park SM, Chatterjee VKK. Genetics of congenital hypothyroidism. J Med Genet, 2005; 42: 379-389.

10. Adeniran KA, Limbe M. Review article on congenital hypothyroidism and newborn screening program in Africa; The present situation and the way forward. Thyroid Disorders Ther, 2012; 1: 1.

11. Ahmad N, Irfan A, Saedi SAA. Congenital hypothyroidism: Screening, diagnosis, management, and outcome. J Clin Neonatol, 2017; 6: 64-70.

12. International Atomic Energy Agency. Screening of newborns for congenital hypothyroidism, guidance for developing programmes. 2005; 12-65.

13. Langham S, Hindmarsh $P$, Krywawych $S$, Peters C. Screening for congenital hypothyroidism: Comparison of borderline screening cut-off points and the effect on the number of children treated with Levothyroxine. Eur Thyroid J, 2013; 2: 180-186.

14. Mandel SJ, Hermos RJ, Larson CA, Prigozhin AB, Rojas DA, Mitchell ML. Atypical hypothyroidism and the very low birthweight infant. Thyroid, 2000; 10(8): 693-695.

15. Bhattacharya A. Congenital hypothyroidism and developmental difficulties. Gen Med (Los Angel), 2013; 1 : 1 .

16. Bakopoulos N, Despotidis O, Saridi M. Congenital hypothyroidism: A variety of clinical and mental signs. International Journal of Caring Sciences, 2015; 8(3): 819-829.

17. Klein $A H$, Meltzer S, Kenny FM. Improved prognosis in congenital hypothyroidism treated before age three months. JPediatr, 1972; 81(5): 912-915.

18. LaFranchi SH, Austin J. How should we be treating children with congenital hypothyroidism?. JPediatrEndocrinol, 2007; 20(5): 559-578. 